\title{
Desafios da enfermagem em epidemiologia da Covid-19: um estudo bibliométrico
}

\author{
Nursing challenges in Covid-19 epidemiology: a bibliometric study \\ Desafíos de enfermería en la epidemiología del Covid-19: un estudio bibliométrico
}

Recebido: 23/09/2021 | Revisado: 30/09/2021 | Aceito: 09/10/2021 | Publicado: 10/10/2021

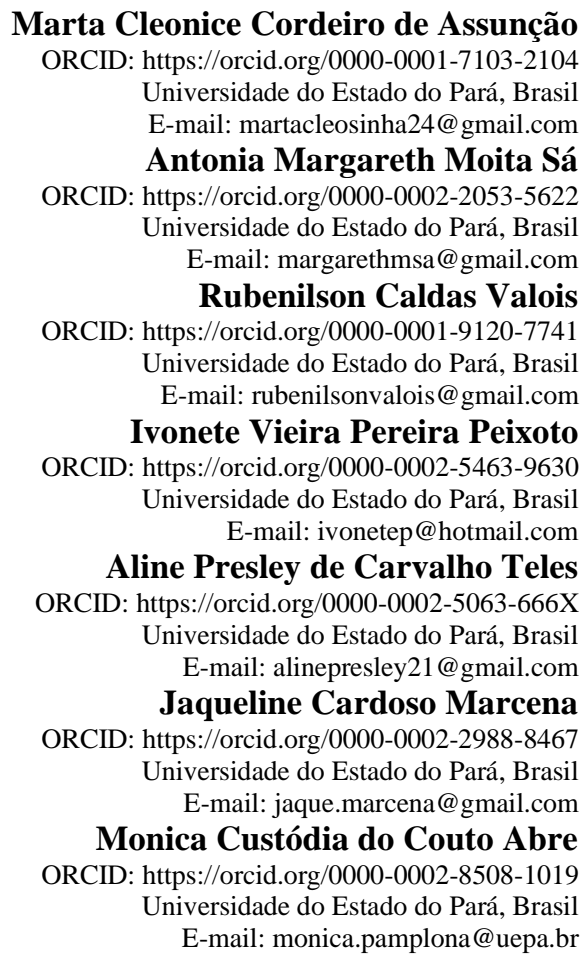

\begin{abstract}
Resumo
Considerando a importância dos profissionais de enfermagem na organização e gestão do cuidado, objetivou-se com este estudo mapear a produção científica mundial em uma base de dados sobre estudos que relatam possíveis desafios da enfermagem atuante em epidemiologia no enfrentamento da Covid-19, tendo por base o protagonismo de autores brasileiros. Estudo descritivo, do tipo bibliométrico extraído da base Web of Science, com banco de dados organizado no Microsoft Excel 2019 e análise por meio do software VOSviewer® (versão 1.6.16). Foram encontrados 231 artigos (78, 5\%) originais e $63(21,5 \%)$ artigos de revisão com predomínio dos United State of América (27,8\%). Os periódicos compõem o estrado A da Capes, em quase sua totalidade. A produção brasileira da temática em questão quando comparada às publicações de outros países mostrou-se insuficiente para o atendimento das demandas atuais das atividades de enfermagem em epidemiologia. Em síntese, um relevante volume de publicações que contemplam o aspecto epidemiológico centralizado nos processos assistenciais do enfermeiro e baixo volume sobre ações específicas de atuação da enfermagem em epidemiologia.
\end{abstract}

Palavras-chave: Enfermagem; Epidemiologia; Infecção por coronavírus.

\begin{abstract}
Considering the importance of nursing professionals in the organization and management of care, the aim of this study was to map the scientific production in a database on studies reporting possible challenges of nursing working in epidemiology in coping with Covid-19, having based on the protagonism of Brazilian authors. Descriptive bibliometric study extracted from the Web of Science database, with a database organized in Microsoft Excel 2019 and analysis using the VOSviewer ${ }^{\circledR}$ software (version 1.6.16). 231 original articles (78.5\%) and 63 (21.5\%) review articles were found, with a predominance of the United State of America (27.8). The periodicals make up the A stage of Capes, in almost its entirety. The Brazilian production of the subject in question, when compared to publications from other countries, proved to be insufficient to meet the current demands of nursing activities in epidemiology. In summary, a relevant volume of publications that contemplate the epidemiological aspect centered on the care processes of nurses and a low volume on specific actions of nursing performance in epidemiology.
\end{abstract}

Keywords: Nursing; Epidemiology; Infection from coronavirus. 


\begin{abstract}
Resumen
Considerando la importancia de los profesionales de enfermería en la organización y gestión del cuidado, el objetivo de este estudio fue mapear la producción científica mundial en una base de datos sobre estudios que reportan los posibles desafíos de la enfermería que trabaja en epidemiología en el afrontamiento del Covid-19, teniendo como base sobre el protagonismo de los autores brasileños. Estudio bibliométrico descriptivo extraído de la base de datos Web of Science, con base de datos organizada en Microsoft Excel 2019 y análisis mediante software VOSviewer® (versión 1.6.16). Se encontraron 231 artículos $(78,5 \%)$ originales y 63 (21,5\%) artículos de revisión, con predominio de los Estados Unidos de América (27,8\%). Las publicaciones periódicas componen la etapa A de Capes, casi en su totalidad. La producción brasileña del tema en cuestión, en comparación con publicaciones de otros países, resultó ser insuficiente para satisfacer las demandas actuales de las actividades de enfermería en epidemiología. En resumen, un volumen relevante de publicaciones que contemplan el aspecto epidemiológico centrado en los procesos de atención al enfermero y un volumen bajo en acciones específicas del desempeño de enfermería en epidemiología.
\end{abstract}

Palabras clave: Enfermería; Epidemiología; Infección por coronavirus.

\title{
1. Introdução
}

Em 31 de dezembro de 2019, a Organização Mundial da Saúde (OMS) foi alertada sobre vários casos de pneumonia na cidade de Wuhan, província de Hubei, na República Popular da China. Referia-se a uma nova cepa (tipo) de coronavírus identificada em 7 de janeiro de 2020 pelas autoridades chinesas e em 30 de janeiro do mesmo ano, a OMS declarou que o surto do novo coronavírus constitui uma Emergência de Saúde Pública de Importância Internacional (ESPII), o mais alto nível de alerta da Organização (PAHO, 2020).

A relevância social da Covid-19 é evidente e mantém sua representatividade epidemiológica como agravo que comporta em seus registros oficiais até o momento (26 de junho de 2021), o quantitativo acumulado de 178.837 .204 casos confirmados e 3.880.450 no mundo, notificados à OMS. A Região das Américas apresentou o maior número de casos acumulados (OMS, 2020). O Brasil, no mesmo período, atingiu um total de 18.169.881 infectados e 507.109 mortes (taxa de letalidade em 2,8\%), representando o segundo maior número de casos acumulados entre os países da região das Américas (WHO, 2020; Brasil, 2021).

Os profissionais de saúde são grandes aliados no combate às epidemias ocorridas entre os séculos, especialmente os profissionais de enfermagem, protagonistas na organização e gestão do cuidado, bem como no combate e controle da propagação da COVID-19 (Backes et al. 2020). A vigilância reforçada dos casos de COVID-19 foi implementada na França em 10 de janeiro de 2020. A adoção de medidas de controle, incluindo quarentena domiciliar para aqueles avaliados com risco moderado / alto de exposição, diminuem o risco de transmissão de pessoa para pessoa originada de casos importados e subsequentemente atrasa propagação do vírus na população em geral (Sibylle, 2020). De que maneira os desafios vivenciados pelos profissionais de saúde sobre o enfrentamento da covid-19 influenciam as ações do profissional de enfermagem que atua na epidemiologia?

Este estudo tem por objetivo mapear a produção científica em uma base de dados internacional sobre estudos que relatam possíveis desafios que refletem nas ações de enfermagem em epidemiologia no enfrentamento da Covid-19 e outros coronavírus entre os anos de 2018 a 2021, ressaltando a representatividade destes profissionais nos serviços de promoção, prevenção e vigilância em saúde nos serviços de saúde públicos e privados.

\section{Metodologia}

Trata-se de um estudo com abordagem quantitativa, do tipo bibliométrico que, se propõe a mensurar o volume das produções científicas internacionais e seu impacto na base de dados Wen of Science. A bibliometria tem como proposta quantificar a produção e a comunicação científica com o escopo de difundir publicações, produtividade de autores e instituições, com o objetivo de evidenciar o crescimento da ciência e o impacto de publicações diante do cenário internacional (Hutchins, 2016; Costa et al. 2020). 
A bibliometria surgiu no início do século XX mediante a necessidade avaliação das produções e comunicação científica deste período histórico. Sua composição abrange três leis, as quais: contemplam a dispersão mediante a medição da produtividade dos periódicos e estabelecer o núcleo e áreas de dispersão sobre um dado assunto (Lei de Bradford); descrevem o perfil das produções científicas dos autores, onde, em sua maioria poucos autores produzem muito, representam uma certa autoridade (referência) sobre uma determinada temática e muitos autores produzem pouco (Lei de Lotka); desenvolve-se o princípio do menor esforço, que consiste na economia de palavras, para que elas não se dispersem, mensurando a ocorrência de palavras que estão presentes no documento proposta pela Lei de Zipf (Tavares; Celerino, 2018; Maia, 2020).

A pergunta norteadora teve por base a estratégia PICo (P: Paciente; I: Intervenção; Co: Contexto) "quais os possíveis desafios enfrentados no mundo pelos profissionais de saúde com relevância no processo de trabalho da enfermagem que atua na vigilância epidemiologia da COVID-19 e outros coronavírus entre os anos de 2018 a 2021 ?

A primeira etapa da estratégia de busca abrangeu a escolha dos DeCS/MeSH (Descritores em Ciências da Saúde) "Infecção por coronavírus, epidemiologia e enfermagem" e seus referentes termos em inglês, associados aos termos booleanos AND e OR com inclusão de termos alternativos, com o propósito de ampliar a quantidade de publicações, aplicada posteriormente à base de dados da Web of Science.

$\mathrm{Na}$ etapa posterior, foram estabelecidos os critérios de inclusão a seguir: artigos originais, texto completo e indexado na base de dados, protocolos, artigos de revisões e originais que abordem a temática, publicações entre os anos de 2018 a 2021, idiomas português, inglês e espanhol; e como critérios de exclusão: cartas ao editor, teses, dissertações, textos de acesso restrito e inacessível.

Em relação a aplicação dos filtros para seleção dos periódicos, a primeira busca ofertou 327 publicações e após os refinamentos quanto ao ano de publicação, materiais de acesso aberto e tendo por base os critérios predeterminados, em que, proveio a amostra final de 294 publicações. A exportação e edição dos dados retirados da base de dados foram condensadas e finalizadas através do Microsoft Excel 2019 por intermédio de gráficos.

A análise descritiva ocorreu por meio do software VOSviewer® (versão 1.6.16) resultou na rede de co-ocorrência das palavras-chaves da pesquisa atendendo a lei de Zipf.

\section{Resultados e Discussão}

Em 2018 o artigo selecionado aborda a experiência de enfermeiras no cuidado ao paciente com MERS-CoV, de grande relevância ao contexto da enfermagem que atua na vigilância da Covid-19 (por sua semelhança na dinâmica dos vírus), no que tange a utilidade das estratégias de rastreamento para enfrentamento de surtos de doenças infecciosas. O monitoramento de casos suspeitos, confirmados e acompanhamento dos seus respectivos contatos foram comuns nas epidemias anteriores, partindo do princípio de bloquear a cadeia de transmissão da doença. No total foram encontradas 294 publicações, de acordo com o Gráfico 1. 
Gráfico 1: Frequência de publicações na Web of Science distribuídas entre os anos de 2018 a 2021.

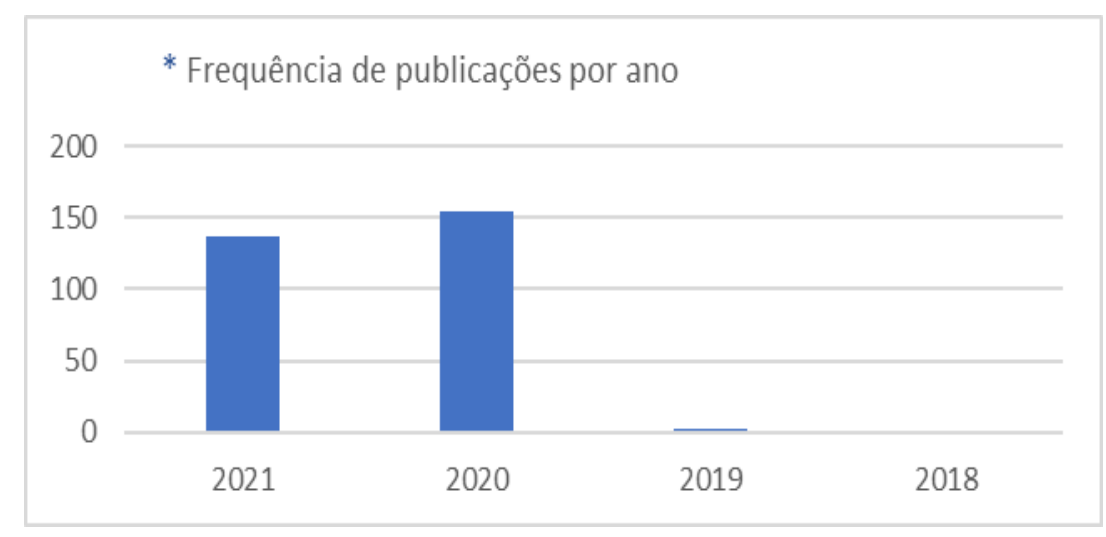

Fonte: autores da pesquisa, (2021), Belém-Pará, Brasil.

Quanto ao tipo de documento foram encontrados 231 artigos (78,5\%) originais e 63 (21,5\%) artigos de revisão. Em relação a distribuição das publicações por país o Gráfico 2 aponta o predomínio de United States of América (USA), representando $82(27,8 \%)$ registros e o Brasil soma 15 publicações (5\%) entre os países, motivo de instigação para o desenvolvimento de novas pesquisas sobre a temática proposta.

Gráfico 2: Distribuição das publicações de acordo com os países de origem sobre a temática entre os anos de 2018 a 2021 , Belém-Pará, Brasil, 2021.

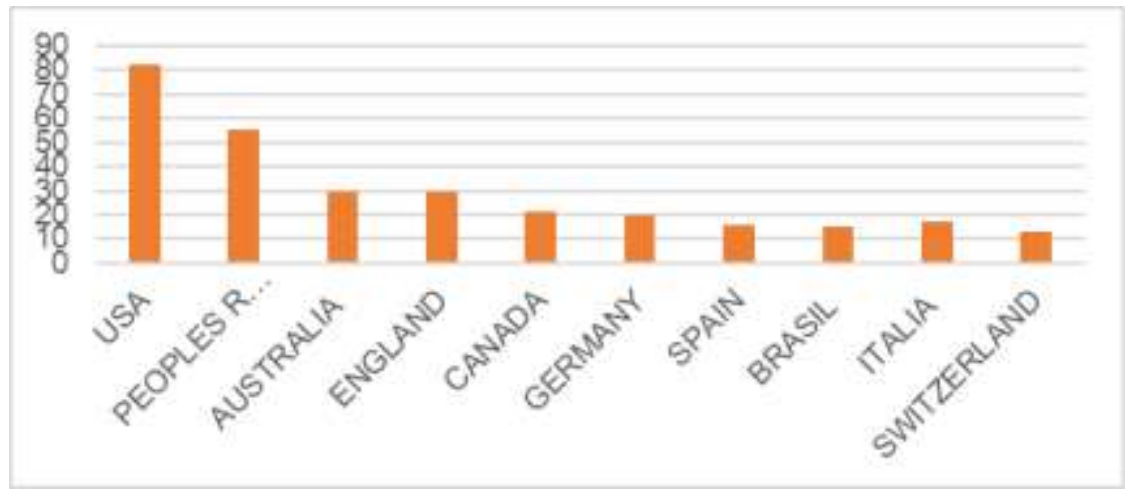

Fonte: autores da pesquisa, (2021), Belém-Pará, Brasil.

O idioma de publicação segue na predominância do inglês 285 (97\%), alemão 4 (1\%), espanhol 3 (1\%), francês 1 $(0,5 \%)$ e português $1(0,5 \%)$.

Reportando-se à lei de Bradford (grau de relevância dos periódicos), foi constatado que o periódico American Journal of Infection Control apresentou 20 publicações. Quanto a representatividade das produções contemplou 191 periódicos, e elencamos os seguintes achados: núcleo 16 revistas (8\%) que mais publicaram sobre a temática, zona 1 compete a 78 (41\%) revistas e zona 2 com 97 (51\%) revistas. Esta classificação em zonas segue de acordo com Junior et al (2014), pois nestas zonas observa-se o aumento do número de periódicos que reduzem a produtividade de publicação de artigos do respectivo assunto. Em referência a Qualis (2013-2016), foi constatado que a maioria das revistas que compõem o núcleo correspondem ao estrato A, em concordância com o Quadro 1 abaixo. 
Quadro 1: Distribuição de publicações internacionais sobre o tema por periódico e QUALIS entre aos anos de 2018-2021. Belém-Pará, Brasil, 2021. Lei de Bradford.

\begin{tabular}{|c|c|c|}
\hline \multicolumn{3}{|l|}{ Publicações $n=294$} \\
\hline PERIÓDICOS & $\mathrm{f}$ & QUALIS \\
\hline AMERICAN JOURNAL OF INFECTION CONTROL & 20 & A2 \\
\hline $\begin{array}{l}\text { INTERNATIONAL JOURNAL OF ENVIRONMENTAL RESEARCH AND PUBLIC } \\
\text { HEALTH }\end{array}$ & 12 & A2 \\
\hline BMJ OPEN & 11 & A2 \\
\hline EPIDEMIOLOGY AND INFECTION & 9 & A1 \\
\hline INFECTION CONTROL AND HOSPITAL EPIDEMIOLOGY & 7 & A1 \\
\hline PLOS ONE & 5 & A1 \\
\hline ANNALS OF EPIDEMIOLOGY & 4 & A1 \\
\hline BMJ GLOBAL HEALTH & 4 & B2 \\
\hline CLINICAL EPIDEMIOLOGY AND GLOBAL HEALTH & 4 & A1 \\
\hline INTERNATIONAL NURSING REVIEW & 4 & A1 \\
\hline JOURNAL OF CLINICAL NURSING & 4 & A1 \\
\hline BMJ BRITISH MEDICAL JOURNAL & 4 & A1 \\
\hline JOURNAL OF THE AMERICAN MEDICAL DIRECTORS ASSOCIATION & 3 & A1 \\
\hline SOCIAL PSYCHIATRY AND PSYCHIATRIC EPIDEMIOLOGY & 3 & A1 \\
\hline AMERICAN JOURNAL OF EPIDEMIOLOGY & 2 & A1 \\
\hline ANNALS OF TRANSLATIONAL MEDICINE & 2 & B2 \\
\hline
\end{tabular}

Fonte: autores da pesquisa, (2021), Belém-Pará, Brasil.

O quantitativo entre as publicações alcançou um total de 2.358 autores. A contribuição de $20 \%$ (472) da frequência dos autores foram responsáveis por $20,9 \%$ das produções científicas.

No que se refere a lei de Lotka, destacamos o predomínio de autores com pesquisas desenvolvidas em âmbito internacional sobre o tema, apresentando uma equiparação numérica de publicações de acordo com o Gráfico 3. Em consonância com Junior et al (2014) a produção deste número reduzido de autores se iguala em quantidade ao desempenho de muitos autores que possuem baixo volume de publicação.

Coutinho (1991) corrobora que este núcleo é a zona de produtividade número 1 da distribuição (núcleo). Os outros periódicos menos produtivos são ordenados em zonas de produtividade decrescente de artigos sobre o mesmo assunto, e o número de periódicos em cada zona aumenta à medida em que sua produtividade individual diminui. A outra lei da bibliometria conhecida como Lei de Lotka ou lei do quadrado inverso aborda a produtividade dos autores (Coutinho, 1991), conforme o Gráfico 3. 
Gráfico 3: Autores com maior quantitativo de publicações sobre o tema entre os anos de 2018-2021, Belém-Pará, Brasil. Lei de Lotka.

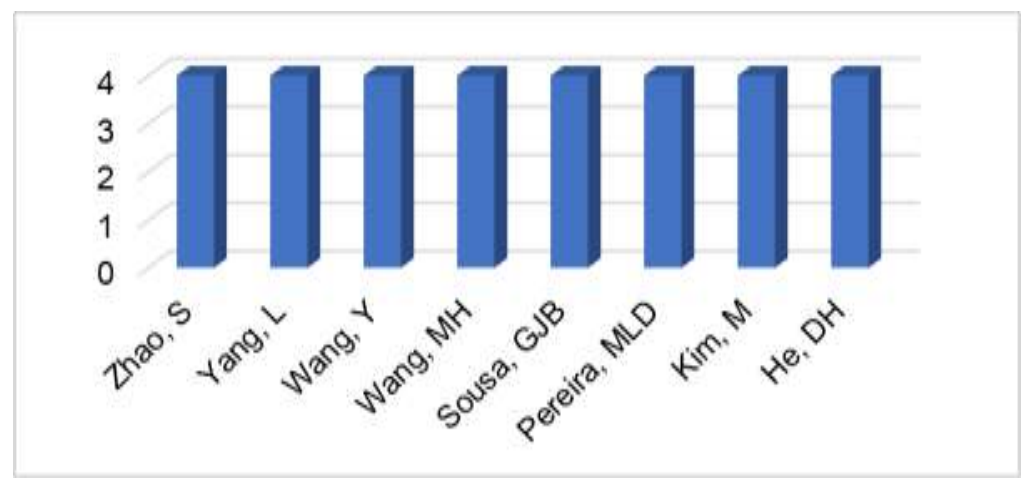

Fonte: autores da pesquisa, (2021), Belém-Pará, Brasil.

Quanto à Lei de Zipf também conhecida como a lei do mínimo esforço avalia a frequência das palavras que mais aparecem entre as publicações (Ribeiro, 2017). A palavra central em destaque nos artigos correspondeu a: Covid-19, a qual, foi mencionada 158 vezes nas publicações entre um somatório de 1359 palavras. As zonas integrantes desta lei quantificam as palavras que mais se repetem entre os textos. A zona trivial integra $7(25,4 \%)$ palavras, zona de interesse $124(29,1 \%)$ palavras e zona de ruídos $618(45,5 \%)$ palavras. A Figura 1 aponta a co-ocorrência das palavras-chaves que compõem a zona trivial (maior frequência).

Figura 1: Rede de co-ocorrência das palavras-chaves que compõem a zona trivial dos estudos selecionados operacionalizados no software VOSviewer®, Belém-PA, Brasil, 2021. Lei de Zipf.

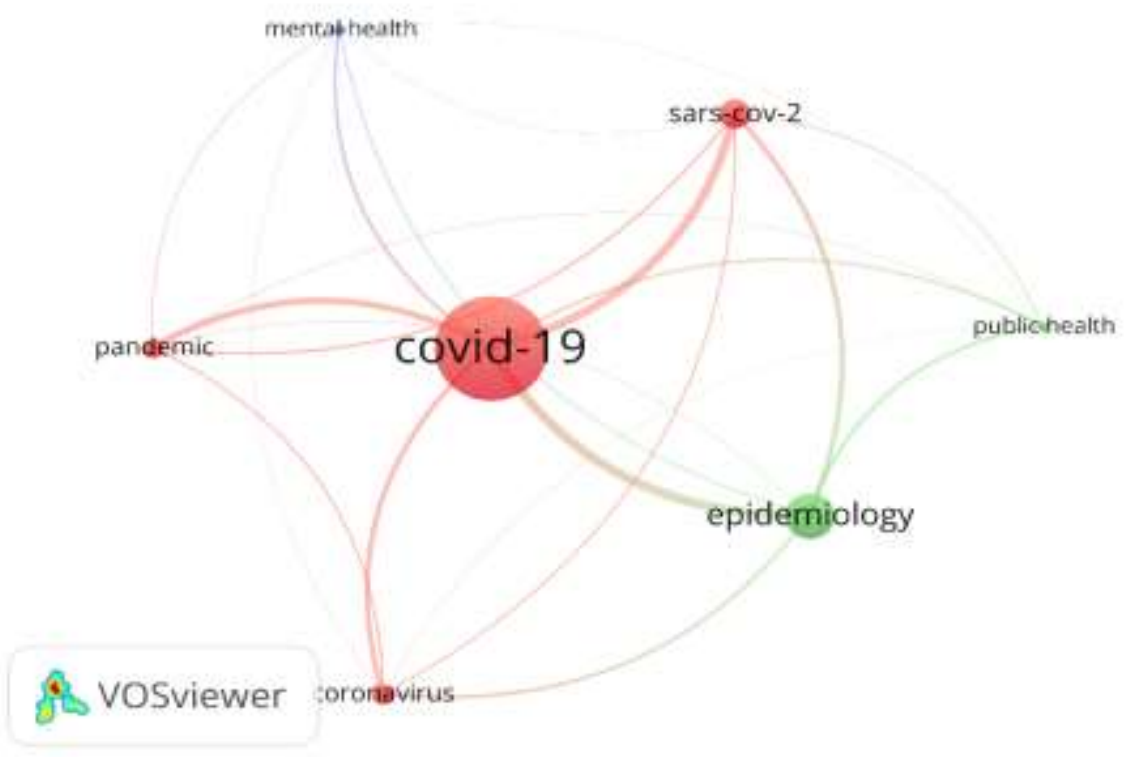

Fonte: Autores da pesquisa, (2021), Belém-Pará, Brasil.

A Figura 1 apresenta-nos a rede de co-ocorrência das palavras-chaves que compões a zona trivial caracterizado pela de Lei de Zipf e ilustra a frequência desta ocorrência. A identificação na formação das palavras é representada por meio de nós (pontos de aglomeração denominados cluster) e conectam em redes classificadas em três cores: vermelho, verde e azul. A palavra Covid-19 foi o destaque entre os termos e mostra sua relevância de escolha e interligação com todas as palavras, até as de menos destaque. 
Portanto, observa-se que o conjunto vermelho possui quatro palavras-chave e engloba o termo infecção por coronavírus. O conjunto verde compreende à importância da relação do termo epidemiologia e as ações de saúde pública no combate da Covid-19 no Brasil e mundo. O cluster azul relaciona o impacto negativo da pandemia na saúde mental dos profissionais de saúde e a equipe de enfermagem segue afetada neste quesito, de acordo com a elevada associação desse ponto de aglomeração com o termo enfermagem.

Tornou-se evidente que o ano de 2020 foi marcado por uma intensa produção científica sobre o novo coronavírus e segue na mesma tendência em 2021. Os artigos selecionados no ano 2019 trazem a abordagem da infecção por coronavírus referente à síndrome respiratória do oriente médio (MERS-CoV). Os artigos selecionados entre o período de 2018 e 2019 não abrangem o tempo de disseminação da Covid-19, todavia, foram incluídos neste estudo pela adoção de estratégias de monitoramento até então mantidas na pandemia do Sars-Cov-2 em vários países.

Um estudo realizado pela Universidade de Harvard avaliou a associação de características sociodemográficas e conhecimento sobre a pandemia da doença do coronavírus entre adultos norte-americanos e constatou a necessidade imediata de aumentar as informações precisas e encorajar comportamentos apropriados entre as comunidades minoritárias, homens e jovens (Alsan, et al. 2020). O processo de educação em saúde faz parte do processo de trabalho dos profissionais de saúde e o enfermeiro desenvolve-o junto de sua equipe de acordo com o cenário epidemiológico atual, o qual, propõe aplicação de métodos resolutivos para cada caso, suspeito ou confirmado de infecção pelo Sars-Cov-2.

A Covid-19 é uma enfermidade sem tratamento farmacológico específico comprovado e validado (Figueiredo et al. 2020). Sua transmissibilidade e letalidade seguem com muitas lacunas de caráter clínico-epidemiológico dificultando a implementação de intervenções efetivas dos serviços de saúde, constituindo um desafio atual e vindouro aos profissionais de enfermagem que seguem diretamente afetados na linha de frente dos serviços de urgência, cuidados críticos, saúde pública, epidemiologia e demais locais com elevado potencial de disseminação. Desafios que englobam a qualidade de saúde mental frente ao adoecimento alheio, ausência de protocolos epidemiológicos implantados nos ambientes de trabalho, ausência de tratamento específico nos casos leves e graves, sobrecarga de trabalho ocasionado dimensionamento deficiente da equipe, lidar com a morte e o luto podem constituir algumas limitações que afetam as atividades hospitalares em epidemiologia.

Estratégias de enfrentamento pessoal e fatores que podem aumentar a motivação da equipe para trabalhar durante eventos futuros de natureza semelhante, também foram questionados em um estudo realizado pelo Hospital de Stamford nos Estados Unidos. Os profissionais de saúde correm o risco elevado de contrair a doença em questão pelo seu alto grau de disseminação entre indivíduos sintomáticos e assintomáticos. Os estudos que avaliam o impacto psicológico entre profissionais de enfermagem que atendem pacientes hospitalizados na pandemia ainda são limitados (Rose; Hartnett; Pillai. 2021).

Sabe-se que o coronavírus integrou outros agravos respiratórios anteriores à pandemia da Covid-19 a exemplo da Síndrome Respiratória do Oriente Médio transmitida por camelos dromedários (MERS-CoV) transmitida dos animais para humanos e entre humanos (Gardner, 2019). A suscetibilidade de uma população à infecção possui relação com a capacidade que os sistemas de saúde identificam os portadores do vírus (sintomáticos e assintomáticos) impactando de forma relevante ou não em sua incidência. Esta capacidade de identificação dos pacientes ocorre por ações estratégicas de rastreamento (assintomáticos) e monitoramento (sintomáticos) e falta de identificação dos casos leves aumentará a magnitude da letalidade (Figueiredo et al. 2020). Estes fatos corroboram na sobrecarga de atendimento dos serviços de saúde, principalmente em locais mais vulneráveis pela escassez de profissionais e recursos técnicos.

Um estudo da Universidade de Medicina da Coreia em 2019 realizou uma análise retrospectiva de base hospitalar, a qual, comparou pacientes MERS-CoV-positivos e MERS-CoV-negativos. A radiografia de tórax com broncopneumonia difusa foi mais frequentes em pacientes MERS-CoV-positivos do que em pacientes negativos; cefaleia, mialgia e diarreia foram os achados comuns entre os casos positivos (Hwang, 2019). A grande variedade de agentes etiológicos virais com sintomatologia 
semelhante ao SARS-Cov-2 requer estratégias clínicas e laboratoriais atenciosas para diagnóstico diferenciado. Ao compararmos alguns fatores de propagação do atual SARS-Cov-2 e MERS-CoV, o último se espalhou rapidamente na Coreia do Sul dentro de 1 mês após o primeiro diagnóstico, devido ao atendimento familiar, visitas de familiares, compras de hospitais, falta de quartos isolados e salas de emergência superlotadas (Kim, 2018).

Tais características semelhantes aos sintomas atípicos da Covid-19 devem ser levadas em consideração nas atividades de rastreamento e monitoramento, pois estamos vivendo uma pandemia imersa em outras epidemias e endemias e cabe ao profissional enfermeiro e demais profissionais responsáveis atuantes nos serviços de epidemiologia buscar pelas características diferenciais de caráter clínico, laboratorial e epidemiológico.

Descobrimos que buscar aumentar a vigilância de hospedeiros sintomáticos por si só não é normalmente a estratégia ideal para reduzir os riscos de surto. A adoção de uma estratégia que combina um aprimoramento da vigilância de casos sintomáticos com esforços para encontrar e isolar hospedeiros infectados não sintomáticos leva à maior redução na probabilidade de que os casos importados iniciem um surto local (Lovell-Read et al. 2021).

A rápida propagação requer atividades de vigilância constantes e adaptadas ao contexto de uma localidade. Visitas domiciliares são comuns no exercício das atividades da equipe de enfermagem e se torna oportunidade para rastreamento de contatos e solidificação das estratégias de educação em saúde da família e coletividade. O contato próximo do enfermeiro com a comunidade eleva a oportunidade de uma abordagem de vigilância educativa e preventiva, contribuindo de forma não onerosa, levando em consideração a escassez de recursos técnicos em locais de maior vulnerabilidade.

Em um estudo realizado em Fortaleza, no estado do Ceará por Alves, Gomes e Custódio (2021) relatam que no início da pandemia, foi muito difícil trabalhar com uma doença desconhecida e altamente contagiante, principalmente porque o que garantia um pouco de segurança aos profissionais para não se contaminarem estava escasso no mundo. $\mathrm{O}$ aprendizado rápido para o cuidado seguro, foi indispensável no desenvolvimento das atividades e a educação permanente sensibilizou o quadro profissional da necessidade de adotar medidas simples, já conhecidas, como a higienização das mãos e uso do equipamento de proteção individual (EPI'S) durante o desempenho de suas atividades.

A mudança frequente dos protocolos de controle pode gerar dúvidas e sentimentos de medo de contágio de uma doença recente (Kim, 2018). Um estudo realizado pela Universidade de Oxford sobre intervenções direcionadas a casos não sintomáticos concluiu que a adoção de uma estratégia que combina um aprimoramento de casos sintomáticos associados à busca para encontrar e isolar hospedeiros infectados assintomáticos leva à maior redução na probabilidade de que os casos importados ao iniciar um surto local (Lovell-Read et al. 2021).

A orientação e acompanhamento das atividades de vigilância hospitalar e ambulatorial, urgência e emergência e serviços de epidemiologia devem seguir os protocolos e diretrizes nacionais e locais adaptando-os de acordo com sua realidade do serviço. As experiências adquiridas das epidemias anteriores proporcionaram um aperfeiçoamento no processo epidemiológico mundial e o enfermeiro segue deixando suas contribuições no desenvolvimento de estratégias de educação em saúde em contextos laborais diferenciados no enfrentamento da Covid-19, mesmo enfrentando contextos de estresse, ansiedade, medo e desafio em manter a qualidade dos processos epidemiológicos e reafirma o significado da importância deste profissional na equipe de enfermagem e multiprofissional.

É importante destacar que potenciais restrições de tempo e prioridades concorrentes são barreiras para a capacidade dos enfermeiros de fazer uma coleta robusta de literatura e avaliações de evidências para implementar em sua assistência (Loresto Jr et al. 2021). Ao decorrer deste estudo não foi detectado um protocolo ou diretriz específica da categoria de enfermagem que orientem sua atuação em vigilância epidemiologia no enfrentamento da Covid-19. A ausência de orientações técnicas direcionadas à equipe de enfermagem constitui um grande desafio destes profissionais quanto ao respaldo técnicocientífico e direcionamento das atividades aos profissionais que atuam em diferentes contextos da vigilância epidemiológica. 
A produção brasileira da temática em questão quando comparada às publicações de outros países é insuficiente para o atendimento das demandas atuais das atividades de enfermagem em epidemiologia. Este achado propõem a instigação quanto aos desafios pela busca de novos estudos que abordem o protagonismo da enfermagem nas ações de epidemiologia da Covid19. A centralização de publicações no impacto psicológico da equipe de enfermagem, centralização dos cuidados intensivos, de urgência e emergência são evidentes e marcantes no volume de publicações da base de dados analisada.

\section{Conclusão}

Constatou-se um relevante volume de publicação que contempla o aspecto epidemiológico centralizado nos processos assistenciais do enfermeiro, com amplo direcionamento relacionado às práticas curativas de enfermagem e um baixo volume publicado sobre epidemiologia com protagonismo de atuação do profissional enfermeiro, mas atende aos critérios de inclusão da enfermagem na equipe multiprofissional na realização das atividades em saúde pública e epidemiologia em geral.

Em síntese, no decorrer dos achados da literatura a enfermagem é uma ciência capaz de atuar em vários cenários de saúde com dedicação de seus cuidados ao ser humano em sua individualidade e coletividade. Segue sua atuação profissional de maneira representativa nos serviços de saúde do país, mas com um olhar assistencial. Novas propostas de estudos que abordem descrições detalhadas sobre os processos de vigilância epidemiológica da Covid-19 direcionada aos profissionais de enfermagem são necessárias para ampliar o respaldo técnico-científico desta categoria nas atividades de epidemiologia.

As atividades de epidemiologia relacionadas à Covid-19 são marcadas por desafios de dimensionamento profissional, dinâmica viral desconhecida, adoecimento da equipe, ausência e/ou precariedade de protocolos técnicos adaptados ao contexto de populações mais vulneráveis. A identificação do baixo volume sobre a temática de atuação da enfermagem em epidemiologia da Covid-19 dificulta o processo de inovação do conhecimento técnico-científico, elaboração de novos protocolos e estratégias direcionadas aos serviços de enfermagem que atuam no setor de epidemiologia, que possam contribuir no processo de saúde pública no que tange ao enfrentamento da Covid-19.

\section{Referências}

Alves, A. R., Gomes, I. L. V., \& Custodio, L. L. Educação permanente em enfermagem na COVID-19: Relato de experiência. Cadernos ESP, Ceará. 2021, JAN-ABR.; 15(2):Suplemento Único. https://cadernos.esp.ce.gov.br/index.php/cadernos/article/view/534/271

Alsan, M., Stantcheva, S., Yang, D., \& Cutler, D. Disparities in Coronavirus 2019 Reported Incidence, Knowledge, and Behavior Among US Adults. JAMA Network Open. 2020;3(6):e2012403. 10.1001/jamanetworkopen.2020.12403

Backes, M. T. S., et al. New coronavírus: what does nursing have to learn and teach in times of a pandemic? Rev Bras Enferm. 2020;73(Suppl 2):e20200259. doi: http://dx.doi.org/10.1590/0034-7167-2020-0259. https://www.scielo.br/j/reben/a/8gnFNHnp36W6mWBJzHqWzYb/?lang=en\&format=pdf

Bernard Stoecklin Sibylle e col. First cases of coronavirus disease 2019 (COVID-19) in France: surveillance, investigations and control measures, January, 2020. EuroSurveill. 2020;25(6):pii=2000094. https://doi.org/10.2807/1560-7917.ES.2020.25.6.2000094 https://www.eurosurveillance.org/content/10.2807/ 1560-7917.ES.2020.25.6.2000094\#html_fulltext]

Costa, I. C. P., Sampaio, R. S., Souza, F. A. C., Dias, T. K. C., Costa, B. H. S., \& Chaves, E. C. L. Produção científica em periódicos online sobre o novo coronavírus (COVID-19): pesquisa bibliométrica. Texto Contexto Enferm [Internet]. 2020 [acesso MÊS ANO DIA]; 29:e20200235. https://doi.org/10.1590/1980-265X-TCE-2020-0235

Coutinho, E. Aplicação da lei de Bradford à literatura técnica sobre ferrovia: análise de periódicos e avaliação da base de dados da Rede Ferroviária Federal S.A. Ci. Inf., Brasília, 20(2): 169-180,jul./dez. 1991[internet]. https://www.brapci.inf.br/_repositorio/2011/05/pdf_1e4d794979_0016745.pdf

Figueiredo, A. M., et al. Letalidad de la COVID-19: ausencia de patrón epidemiológico. Gac Sanit. (2021);35(4):355-357. Disponível em: https://doi.org/10.1016/j.gaceta.2020.04.001

Gardner, E. G., et al (2019). Force of infection of Middle East respiratory syndrome in dromedary camels in Kenya. Epidemiology and Infection 147, e275, 16. https://doi.org/10.1017/S0950268819001663

Hutchins, B. I., Yuan, X., Anderson, J. M., \& Santangelo, G. M. Relative Citation Ratio (RCR): a new metric that uses citation rates to measure influence at the article level. PLoS Biol. [Internet] 2016 [acesso 2020 Abr 14];14(9):e1002541. https://doi.org/10.1371/journal.pbio.1002541

Hwang, S. M., et al. Clinical and Laboratory Findings of Middle East Respiratory Syndrome Coronavirus Infection. Jpn. J. Infect. Dis., 72, 160-167, 2019. 10.7883/yoken.JJID.2018.187 
Research, Society and Development, v. 10, n. 13, e251101320988, 2021

(CC BY 4.0) | ISSN 2525-3409 | DOI: http://dx.doi.org/10.33448/rsd-v10i13.20988

Junior, C. M., et al. Análise de Viabilidade de Utilizar as Leis da Bibliometria em Diferentes Bases de Pesquisa. Associação Nacional de Pós-Graduação e Pesquisa em Administração - AMPAD- Rio de Janeiro 13 a 17 de setembro de 2014 [internet]. http://anpad.org.br/admin/pdf/2014_EnANPAD_EPQ762.pdf

Kim, Y. Nurses' experiences of care for patients with Middle East respiratory syndrome-coronavirus in South Korea. Am J Infect Control. 2018 Jul;46(7):781787. 10.1016/j.ajic.2018.01.012.

Lovell-Read, F. A., Funk, S., Obolski, U., \& Donnelly, C. A. Thompson RN. 2021 Interventions targeting non-symptomatic cases can be important to prevent local outbreaks: SARS-CoV-2 as a case study. J. R. Soc. Interface 18: 20201014. https://doi.org/10.1098/rsif.2020.1014

Loresto Jr, F. L., et al. The nurse COVID and historical epidemics literature repository: Development, description, and summary. Nurs Outlook 69 (2021) 257 264. 10.1016 / j.outlook.2020.12.017

Maia, M., \& Bezerra, C. A. Análise bibliométrica dos artigos científicos de jurimetria publicados no Brasil. RDBCI: Rev. Digit. Bibliotecon. Cienc. Inf. [Internet]. $30^{\circ}$ de junho de 2020 [citado $29^{\circ}$ de junho de 2021];18(00):e020018. https://periodicos.sbu.unicamp.br/ojs/index.php/rdbci/article/view/8658889

Ministério da Saúde. Secretaria de Vigilância em Saúde. Departamento de informação do SUS. Painel de casos pelo coronavírus, 2021. https://covid.saude.gov.br/

Pan American Health Organization (PAHO). Histórico da Pandemia de Covid-19. PAHO/WHO, 2020 [acesso em 26 jun 2021]. https://www.paho.org/pt/covid19/historico-da-pandemia-covid-19

Rose, S., Hartnett, J., \& Pillai, S. Healthcare worker's emotions, perceived stressors and coping mechanisms during the COVID-19 pandemic. PLoS ONE 16(7): e0254252, 2021. https://doi. org/10.1371/journal.pone.0254252

Ribeiro, H. C. M. Bibliometria: quinze anos de análise da produção acadêmica em periódicos brasileiros. Universidade Federal do Piauí - UFPI, Brasil [internet], 2017. 10.5195/biblios.2017.39

Tavares, W. Q. T., \& Celerino, V. G. A Importância da Bibliometria para a Indexação Automática. Revista Biblioteconomia e Ciência da Informação Universidade Federal do Cariri - 4(1), 7-15, jul./dez.,2018. https://periodicos.ufca.edu.br

World Health Organization (WHO). Painel do Coronavírus da OMS (COVID-19), 2021. Painel de emergência da Covid-19 [acesso em 26 jun 2021 ]. https://covid19.who.int/ 